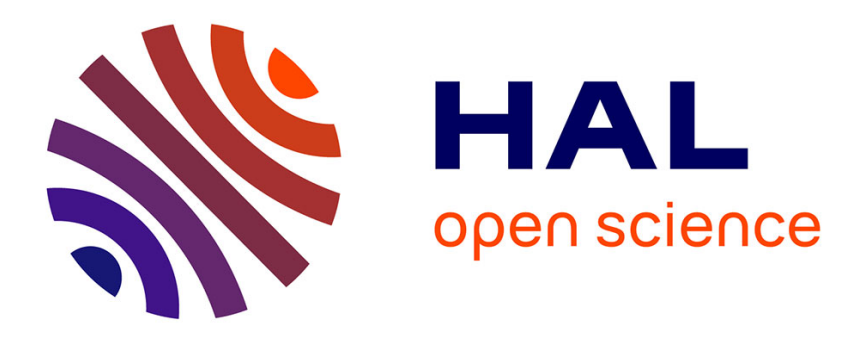

\title{
Uniform-in-Bandwidth Nearest-Neighbor Density Estimation
}

\author{
Sarah Ouadah
}

\section{To cite this version:}

Sarah Ouadah. Uniform-in-Bandwidth Nearest-Neighbor Density Estimation. 2012. hal-00766606

\section{HAL Id: hal-00766606 https://hal.science/hal-00766606}

Preprint submitted on 18 Dec 2012

HAL is a multi-disciplinary open access archive for the deposit and dissemination of scientific research documents, whether they are published or not. The documents may come from teaching and research institutions in France or abroad, or from public or private research centers.
L'archive ouverte pluridisciplinaire HAL, est destinée au dépôt et à la diffusion de documents scientifiques de niveau recherche, publiés ou non, émanant des établissements d'enseignement et de recherche français ou étrangers, des laboratoires publics ou privés. 


\title{
Uniform-in-Bandwidth Nearest-Neighbor Density Estimation
}

\author{
Sarah Ouadah* \\ Laboratoire de Statistique Théorique et Appliquée \\ Université Pierre et Marie Curie-Paris 6 \\ 4 place Jussieu, 75252 Paris Cedex 05, France.
}

Draft version, September 10, 2012

\begin{abstract}
We present a new sharp uniform-in-bandwidth limit law for the nearest-neighbor density estimator. Our result is established in the framework of convergence in probability, and we allow the bandwidth to vary within the complete range for which the estimator is consistent. We provide the explicit value of the asymptotic limiting constant for the sup-norm of the estimator's random error.
\end{abstract}

AMS 2010 Classification: 62G05, 62G07, 62G20,62G30, 60F15, 60F17, 60N01, $60 \mathrm{~N} 02$.

Keywords and phrases: Nonparametric density estimation, nearest-neighbor estimator, uniform quantile process, functional limit laws, convergence in probability.

\section{Introduction and Result}

We are concerned with the nonparametric estimation of the density $f(\cdot)$ of a random variable $[\mathrm{rv}] X \in \mathbb{R}$ by the nearest-neighbor $[\mathrm{NN}]$ method. The NN estimators are motivated as follows (see, e.g., Fix and Hodges [17]). Let $X_{1}, X_{2}, \ldots$ be independent and identically distributed [iid] random copies of $X$, with distribution function [df] $\mathbb{F}(x):=\mathbb{P}(X \leq x)$, for $x \in \mathbb{R}$. Denote the empirical df based upon $X_{1}, \ldots, X_{n}$, by $\mathbb{F}_{n}(x):=n^{-1} \#\left\{X_{i} \leq x\right.$ : $1 \leq i \leq n\}$, for $x \in \mathbb{R}$, where \# stands for cardinality. For each $\lambda>0$ and $x \in \mathbb{R}$, set

$$
R_{n, \lambda}(x):=\inf \left\{h>0: \mathbb{F}_{n}(x+h)-\mathbb{F}_{n}(x) \geq \lambda\right\},
$$

and

$$
R_{\lambda}(x):=\inf \{h>0: \mathbb{F}(x+h)-\mathbb{F}(x) \geq \lambda\},
$$

where we make use of the convention that $\inf _{\emptyset}(\cdot)=\infty$. Since $\mathbb{F}_{n}(\cdot)$ and $\mathbb{F}(\cdot)$ are rightcontinuous, it is readily checked from (1) and (2) that

$$
\mathbb{F}_{n}\left(x+R_{n, \lambda}(x)\right)-\mathbb{F}_{n}(x) \geq \lambda \quad \text { and } \quad \mathbb{F}\left(x+R_{\lambda}(x)\right)-\mathbb{F}(x) \geq \lambda .
$$

This, in turn, entails that $R_{n, \lambda}(x) \in(0, \infty]$ and $R_{\lambda}(x) \in(0, \infty]$ for all $x \in \mathbb{R}$ and $\lambda>0$. When the density $f(t)=\mathbb{F}^{\prime}(t)$ of $X$ exists and is continuous in a neighborhood of $x \in \mathbb{R}$, we have, as $\lambda \downarrow 0$,

$$
M_{\lambda}(x):=\frac{\lambda}{R_{\lambda}(x)} \rightarrow f(x) .
$$

\footnotetext{
*E-mail: sarah.ouadah@upmc.fr
} 
In view of (1) and (2), the usual NN-estimator of $f(x)$ is the empirical counterpart of $M_{\lambda}(x)$ in (3), given by

$$
f_{n, \lambda}(x):=\frac{\lambda}{R_{n, \lambda}(x)} .
$$

The convention that $\lambda / \infty=0$ for $\lambda>0$ renders meaningful the definitions of $M_{\lambda}(x) \in$ $[0, \infty)$ in $(3)$, and of $f_{n, \lambda}(x) \in[0, \infty)$ in (4), for all $x \in \mathbb{R}$ and $\lambda>0$. Variants of the NN-estimator $f_{n, \lambda}(\cdot)$ in (4) have been discussed by a number of authors, among whom we should cite Loftsgaarden and Quesenberry [18], Devroye and Wagner [14], Csörgö and Révész [5], Mack [19], Deheuvels and Mason [9], and Viallon [23]. Let $I:=[C, D] \subset J:=$ $[A, B]$, with $-\infty<A<C<D<B<\infty$, be specified sub-intervals of $\mathbb{R}$, under the assumption $(H)$ that $f(\cdot)$ is continuous and strictly positive on $J$. Set $\log _{+} u:=\log (u \vee e)$ for $u \in \mathbb{R}$. Our main result is the following uniform-in-bandwidth convergence theorem.

Theorem 1 Let $0<a_{n} \leq b_{n} \leq 1$ be such that, as $n \rightarrow \infty$,

$$
b_{n} \rightarrow 0 \quad \text { and } n a_{n} / \log n \rightarrow \infty .
$$

Then, with $\mathcal{H}_{n}:=\left[a_{n}, b_{n}\right]$, we have, as $n \rightarrow \infty$,

$$
\sup _{\lambda \in \mathcal{H}_{n}}\left|\left\{\frac{n \lambda}{2 \log _{+}(1 / \lambda)}\right\}^{1 / 2} \sup _{x \in I} \pm\left\{\frac{f_{n, \lambda}(x)-M_{\lambda}(x)}{f(x)}\right\}-1\right|=o_{\mathbb{P}}(1) .
$$

Remark $11^{\circ}$ ) Our proofs will show that the conclusion (6) of Theorem 1 remains valid if, in the definitions (3)-(4) of $M_{\lambda}(x)$ and $f_{n, \lambda}(x)$, we respectively replace $R_{\lambda}(x)$ and $R_{n, \lambda}(x)$, by

and

$$
R_{\lambda}^{*}(x)=\inf \left\{h>0: \mathbb{F}\left(x+\frac{1}{2} h\right)-\mathbb{F}\left(x-\frac{1}{2} h\right) \geq \lambda\right\},
$$

$$
R_{n, \lambda}^{*}(x)=\inf \left\{h>0: \mathbb{F}_{n}\left(x+\frac{1}{2} h\right)-\mathbb{F}_{n}\left(x-\frac{1}{2} h\right) \geq \lambda\right\} .
$$

$\left.2^{\circ}\right)$ It is easy to see that, under (5), the limit law (6) holds with the formal replacement of $\pm\left\{f_{n, \lambda}(x)-M_{\lambda}(x)\right\}$ by $\left|f_{n, \lambda}(x)-M_{\lambda}(x)\right|$.

$\left.3^{\circ}\right)$ The extension of our methods to $N N$ nonparametric estimators of the regression function (see, e.g., Beck [1], Collomb [4], Devroye [13], Burba et al. [3]) will be considered elsewhere.

$\left.4^{\circ}\right)$ The uniform consistency of $f_{n, \lambda}(\cdot)$ over bounded intervals was discussed by Moore and Henrichon [20] (see, e.g., Devroye and Wagner [14]), under the assumption that $f(\cdot)$ is uniformly continuous and positive on $\mathbb{R}$.

$5^{\circ}$ ) Theorem 1 allows us to construct uniform asymptotic simultaneous confidence bands for $f(\cdot)$, in the spirit of that given in Deheuvels and Mason [10] (see, e.g., Deheuvels and Derzko [7], Deheuvels [6]).

As may be checked from our forthcoming proofs, Theorem 1 remains valid if we allow $a_{n}$ and $b_{n}$ to be random and fulfilling (5) in probability. A motivation for uniform-inbandwidth results such as that given in Theorem 1, is to describe the limiting behavior of nonparametric functional estimators when their smoothing parameter (or bandwidth) is possibly random or data-dependent. A number of elaborate schemes have been proposed in the statistical literature for constructing such bandwidth sequences (see, e.g., Berlinet and Devroye [2], and sections 2.4.1-2.4.2 in Deheuvels and Mason [10]). These 
authors typically recommend bandwidths of the form $\lambda=\lambda_{n}^{*}=Z_{n} n^{-1 / 5}$ where $Z_{n}$ is an appropriate (possibly data-dependent) random sequence, stochastically bounded away from 0 and $\infty$. Making use of Theorem 1, we readily obtain a description of the limiting behavior of the corresponding NN-estimators. We refer to Einmahl and Mason [16], and Deheuvels and Ouadah [11], for discussions and references on the closely related problem of uniform-in-bandwidth convergence for kernel estimators. To illustrate the sharpness of (5), we set $\mathcal{H}_{n}=\left[\lambda_{n}, \lambda_{n}\right]$ in Theorem 1 , and observe that, whenever $\left\{\lambda_{n}: n \geq 1\right\}$ are constants fulfilling, as $n \rightarrow \infty$,

$$
\lambda_{n} \rightarrow 0, \quad \text { and } \quad n \lambda_{n} / \log n \rightarrow \infty,
$$

then, as $n \rightarrow \infty$,

$$
\left\{\frac{n \lambda_{n}}{2 \log _{+}\left(1 / \lambda_{n}\right)}\right\}^{1 / 2} \sup _{x \in I} \pm\left\{\frac{f_{n, \lambda_{n}}(x)-M_{\lambda_{n}}(x)}{f(x)}\right\} \stackrel{\mathbb{P}}{\rightarrow} 1 .
$$

Almost sure versions of (8) have been established, under various sets of assumptions, by Csörgö and Révész [5], Mack [19], Deheuvels and Mason [9], and Ralescu [21]. We note that (8) (and hence, (6)), does not hold almost surely [a.s.] for an arbitrary choice of the continuous density $f(\cdot)$ on $J$, and for all bandwidth sequences $\left\{\lambda_{n}: n \geq 1\right\}$ fulfilling (7). If we assume, in addition to (7), that, as $n \rightarrow \infty$,

$$
\log \left(1 / \lambda_{n}\right) / \log \log n \rightarrow \infty, \quad \lambda_{n} \downarrow 0, \quad \text { and } \quad n \lambda_{n} \uparrow \infty,
$$

then (see, e.g., Theorem 4.3 in Deheuvels and Mason [9]), we have, a.s.,

$$
\lim _{n \rightarrow \infty}\left\{\frac{n \lambda_{n}}{2 \log _{+}\left(1 / \lambda_{n}\right)}\right\}^{1 / 2} \sup _{x \in I} \pm\left\{\frac{f_{n, \lambda_{n}}(x)-M_{\lambda_{n}}(x)}{f(x)}\right\}=1 .
$$

This last result is known not to hold in general when the first condition in (9) is not fulfilled. Viallon [23] has obtained a uniform-in-bandwidth convergence theorem in the spirit of (6), by showing that, a.s.,

$$
\lim _{n \rightarrow \infty} \sup _{\lambda \in \mathcal{H}_{n}}\left\{\frac{n \lambda}{2 \log _{+}(1 / \lambda)}\right\}^{1 / 2} \sup _{x \in I}\left|\frac{f_{n, \lambda}(x)-M_{\lambda}(x)}{f(x)}\right|=1,
$$

where $\mathcal{H}_{n}=\left[\lambda_{n}^{\prime}, \lambda_{n}^{\prime \prime}\right]$, and $\lambda_{n}^{\prime}, \lambda_{n}^{\prime \prime}$ are sequences of constants fulfilling (7)-(9) together with at least one of the additional conditions below. As $n \rightarrow \infty$,

$$
\frac{\sqrt{n} \lambda_{n}^{\prime} \log \left(1 / \lambda_{n}^{\prime}\right)}{\log n \sqrt{\log \log \lambda_{n}^{\prime}}} \rightarrow \infty \text { or } \frac{\sqrt{\lambda_{n}^{\prime \prime} \log \left(1 / \lambda_{n}^{\prime \prime}\right)}}{\lambda_{n}^{\prime \prime} \log \left(1 / \lambda_{n}^{\prime \prime}\right)}=o\left(\frac{\sqrt{n}}{\log n}\right) .
$$

We mention that Viallon [23] works under the assumption that $\mathbb{F}(\cdot)$ is twice continuously differentiable on $J$, and such that, for some $\gamma>0$,

$$
\sup _{u \in J} \frac{\mathbb{F}(u)(1-\mathbb{F}(u))\left|f^{\prime}(u)\right|}{f^{2}(u)} \leq \gamma .
$$

Subject to additional conditions on $\mathcal{H}_{n}$, he showed that, as $n \rightarrow \infty$,

$$
\sup _{\lambda \in \mathcal{H}_{n}}\left\{\frac{n \lambda}{2 \log _{+}(1 / \lambda)}\right\}^{1 / 2} \sup _{x \in I}\left|\frac{f_{n, \lambda}(x)-M_{\lambda}(x)}{f(x)}\right| \stackrel{\mathbb{P}}{\rightarrow} 1 .
$$


The conditions imposed on $\lambda_{n}$ in (9), as well as the additional restrictions in Viallon [23], are more strenuous than (5). We should point out that (11) is a much weaker statement than (6). Indeed, the asymptotic limiting constant in (11) relies on a specific $\lambda \in \mathcal{H}_{n}$, whereas the limit law (6) provides the asymptotic limiting constant for the sup-norm of the estimator's random error, uniformly over $\lambda \in \mathcal{H}_{n}$.

The proof of Theorem 1 is postponed until $\S 2.4$. It relies on a uniform-in-bandwidth limit law of Deheuvels and Ouadah [11], which we recall in $\S 2.1$. In $\S 2.2$ and $\S 2.3$, we present preliminaries which are needed in our proofs.

\section{Proofs}

\subsection{A Uniform-in-Bandwidth Limit Law}

The following notation is needed for the statement of the uniform-in-bandwidth limit law of Deheuvels and Ouadah [11] (see Fact 1), which will be instrumental in our proofs. Let $U_{1}, U_{2}, \ldots$ be iid rv's with a uniform distribution on $(0,1)$. Let $\mathbb{U}_{n}(u):=n^{-1} \#\left\{U_{i} \leq u\right.$ : $1 \leq i \leq n\}$, for $u \in \mathbb{R}$, be the empirical df based upon the first $n \geq 1$ of these observations. Define the empirical quantile function [qf] pertaining to $\mathbb{U}_{n}(\cdot)$ by $\mathbb{V}_{n}(v):=\inf \{u \geq 0$ : $\left.\mathbb{U}_{n}(u) \geq v\right\}$, for $0 \leq v \leq 1, \mathbb{V}_{n}(v):=0$ for $v<0$ and $\mathbb{V}_{n}(v):=1$ for $v>1$. Denote the uniform empirical quantile process by

$$
\beta_{n}(u):=n^{1 / 2}\left(\mathbb{V}_{n}(u)-u\right), \text { for } u \in \mathbb{R} .
$$

For each $\mathcal{I}=[r, s] \subseteq[0,1]$, with $r<s$, consider the statistic

$$
\delta_{n, \mathcal{I}}^{ \pm}(h):=\sup _{t \in \mathcal{I} \cap[0,1-h]} \pm\left\{\beta_{n}(t+h)-\beta_{n}(t)\right\} .
$$

Fact 1 is established in Deheuvels and Ouadah [11] (see Corollary 1).

Fact 1 Let $\mathcal{H}_{n}=\left[a_{n}, b_{n}\right]$ be as in Theorem 1. Then, as $n \rightarrow \infty$, we have

$$
\sup _{h \in \mathcal{H}_{n}}\left|\frac{\delta_{n, \mathcal{I}}^{ \pm}(h)}{\sqrt{2 h \log _{+}(1 / h)}}-1\right|=o_{\mathbb{P}}(1) .
$$

Theorem 1 is an application of the uniform-in-bandwidth limit law (14).

\subsection{Some Other Useful Facts}

The following arguments are oriented towards proving Theorem 1 . We inherit the notation of $\S 1$. Let $Q(t):=\inf \{x \in \mathbb{R}: \mathbb{F}(x) \geq t\}$, for $0<t<1$, denote the quantile function [qf] pertaining to $\mathbb{F}(\cdot)$. We extend the definition of $Q(\cdot)$ to the endpoints of $[0,1]$, by setting

$$
Q(0):=A:=\lim _{t \downarrow 0} Q(t), \text { and } Q(1):=B:=\lim _{t \uparrow 1} Q(t) .
$$

We shall provide a proof of Theorem 1 under the assumption $(H)$ that $J=[A, B]$ is the support of $\mathbb{F}(\cdot)$, and that $f(\cdot):=\mathbb{F}^{\prime}(\cdot)$ is continuous and (stictly?) positive on $J$. The extension of our methods to the general case is readily achieved by routine arguments which we omit. The assumption $(H)$ implies that the of $Q(\cdot)$ is differentiable on $[0,1]$, with quantile density $[\mathrm{qd}] q(\cdot):=Q^{\prime}(\cdot)$, continuous and positive on $[0,1]$. We have, namely,

$$
q(t):=\frac{1}{f(Q(t))} \in(0, \infty) \text { for } 0 \leq t \leq 1
$$


Under $(H)$, the rv's $U_{1}:=\mathbb{F}\left(X_{1}\right), U_{2}:=\mathbb{F}\left(X_{2}\right), \ldots$ are uniformly distributed on $(0,1)$, and fulfill, with probability $1, X_{1}=Q\left(U_{1}\right), X_{2}=Q\left(U_{2}\right), \ldots$ We observe that (see, e.g., Proposition 1.1 p. 98 in del Barrio et al. [12])

$$
\mathbb{F}(Q(t))=t, \text { for } 0 \leq t \leq 1, \quad \text { and } \quad Q(\mathbb{F}(x))=x \text {, for } x \in J .
$$

Denote by $Q_{n}(t):=\inf \left\{x \geq 0: \mathbb{F}_{n}(x) \geq t\right\}$, for $0<t<1$, the empirical qf pertaining to $\mathbb{F}_{n}(\cdot)$. Set further

$$
\begin{aligned}
Q_{n}(0) & :=\lim _{t \downarrow 0} Q_{n}(t)=\min \left\{X_{1}, \ldots, X_{n}\right\}, \\
Q_{n}(1) & :=\lim _{t \uparrow 1} Q_{n}(t)=\max \left\{X_{1}, \ldots, X_{n}\right\} .
\end{aligned}
$$

Recalling the definition $\mathbb{U}_{n}(u):=n^{-1} \#\left\{U_{i} \leq u: 1 \leq i \leq n\right\}, u \in \mathbb{R}$, of the uniform empirical df in $\S 2.1$, we recall the definition of the empirical qf based upon $U_{1}, \ldots, U_{n}$, given by

$$
\mathbb{V}_{n}(v):=\inf \left\{u \geq 0: \mathbb{U}_{n}(u) \geq v\right\} \quad \text { for } \quad v \in[0,1] .
$$

We extend the definition of $\mathbb{V}_{n}(\cdot)$ to 0 and 1 , by setting

$$
\mathbb{V}_{n}(0)=\lim _{v \downarrow 0} \mathbb{V}_{n}(v), \quad \mathbb{V}_{n}(1)=\lim _{v \uparrow 1} \mathbb{V}_{n}(v) .
$$

Observe that

$$
Q_{n}(t)=Q\left(\mathbb{V}_{n}(t)\right), \text { for } 0 \leq t \leq 1
$$

and

$$
\mathbb{F}_{n}(x)=\mathbb{U}_{n}(\mathbb{F}(x)), \text { for } x \in \mathbb{R} .
$$

The next fact collects some well-known relations. For (19), refer to (1.6) in Shorack [22], and (2.48)(i) in Fact 6 of Deheuvels and Ouadah [11]. The relation (20) is a consequence of the Dvoretzky, Kiefer and Wolfowitz inequality (see, e.g., Lemma 2 in Dvoretzky et al. [15], and Theorem 3.3 p. 140 in del Barrio et al. [12]). For (21) and (22), we refer to Deheuvels and Devroye [8].

Fact 2 For each fixed $k \geq 1$, we have, as $n \rightarrow \infty$,

$$
\begin{array}{ll}
\text { (i) } & \sup _{0 \leq t \leq 1}\left|\mathbb{F}_{n}\left(Q_{n}(t)\right)-t\right|=n^{-1} \quad \text { a.s.; } \\
\text { (ii) } & \sup _{0 \leq t \leq 1}\left|\beta_{n}(t)\right|=O_{\mathbb{P}}(1) ; \\
\text { (iii) } & \sup _{0 \leq t \leq 1-k / n}\left\{\mathbb{V}_{n}(t+k / n)-\mathbb{V}_{n}(t)\right\}=O_{\mathbb{P}}\left(n^{-1} \log n\right) ; \\
\text { (iv) } & \sup _{0 \leq t \leq 1}\left|\mathbb{V}_{n}\left(\mathbb{U}_{n}(t)\right)-t\right|=O_{\mathbb{P}}\left(n^{-1} \log n\right) .
\end{array}
$$

In (20), we let $\beta_{n}(u)=n^{1 / 2}\left(\mathbb{V}_{n}(u)-u\right), u \in \mathbb{R}$, be as in (12).

\subsection{Proof of Theorem 1 - I}

In this section, we shall assume, unless otherwise specified, that $\mathbb{F}(t)=Q(t)=t$ and $f(t)=q(t)=1$ for $0 \leq t \leq 1$. We shall let throughout $\lambda$ vary in $(0, \infty)$. In view of the definitions (3)-(4) of $M_{\lambda}(\cdot)$ and of $f_{n, \lambda}(\cdot)$, we write,

$$
f_{n, \lambda}(Q(t))-M_{\lambda}(Q(t))=-\frac{\lambda^{2}}{R_{n, \lambda}(Q(t)) R_{\lambda}(Q(t))}\left\{\frac{R_{n, \lambda}(Q(t))-R_{\lambda}(Q(t))}{\lambda}\right\} .
$$

We shall treat the right-hand side of (23) in the next two lemmas. 
Lemma 1 Whenever $\mathcal{H}_{n}=\left[a_{n}, b_{n}\right]$ satisfies (5) in Theorem 1, we have, as $n \rightarrow \infty$,

$$
\sup _{\lambda \in \mathcal{H}_{n}}\left|\left\{\frac{n \lambda}{2 \log _{+}(1 / \lambda)}\right\}^{1 / 2} \sup _{t \in[0,1-\lambda]} \pm\left\{\frac{R_{n, \lambda}(Q(t))-R_{\lambda}(Q(t))}{\lambda}\right\}-1\right|=o_{\mathbb{P}}(1) .
$$

Proof. Since $F(t)=Q(t)=t$ for $0 \leq t \leq 1$, we infer from (17)-(18) that $Q_{n}(t)=\mathbb{V}_{n}(t)$ and $\mathbb{F}_{n}(t)=\mathbb{U}_{n}(t)$ for $0 \leq t \leq 1$. Thus, by (22), we have, as $n \rightarrow \infty$,

$$
\sup _{0 \leq t \leq 1}\left|Q_{n}\left(\mathbb{F}_{n}(t)\right)-t\right|=\sup _{0 \leq t \leq 1}\left|\mathbb{V}_{n}\left(\mathbb{U}_{n}(t)\right)-t\right|=O_{\mathbb{P}}\left(n^{-1} \log n\right) .
$$

By combining the definition (1) of $R_{n, \lambda}(\cdot)$ with (25) and the definition (12) of $\beta_{n}(\cdot)$, we see that, for each $\lambda>0$ and $x \in[0,1]$,

$$
\begin{aligned}
R_{n, \lambda}(x)-\lambda & \left.=\inf \left\{h>0: \mathbb{F}_{n}(x+h)-\mathbb{F}_{n}(x)\right) \geq \lambda\right\}-\lambda \\
& =Q_{n}\left(\mathbb{F}_{n}(x)+\lambda\right)-x-\lambda \\
& =\mathbb{V}_{n}\left(\mathbb{U}_{n}(x)+\lambda\right)-\mathbb{V}_{n}\left(\mathbb{U}_{n}(x)\right)-\lambda+O_{\mathbb{P}}\left(n^{-1} \log n\right) \\
& =n^{-1 / 2}\left\{\beta_{n}\left(\mathbb{U}_{n}(x)+\lambda\right)-\beta_{n}\left(\mathbb{U}_{n}(x)\right)\right\}+O_{\mathbb{P}}\left(n^{-1} \log n\right) .
\end{aligned}
$$

Now, by applying (21), we obtain that, as $n \rightarrow \infty$,

$$
\begin{aligned}
& \sup _{0<\lambda<1}\left|\sup _{0 \leq x \leq 1} \pm n^{-1 / 2}\left\{\beta_{n}\left(\mathbb{U}_{n}(x)+\lambda\right)-\beta_{n}\left(\mathbb{U}_{n}(x)\right)\right\}-\sup _{0 \leq x \leq 1} \pm n^{-1 / 2}\left\{\beta_{n}(x+\lambda)-\beta_{n}(x)\right\}\right| \\
& \leq 2 n^{-1 / 2} \sup _{\substack{0 \leq s, t \leq 1 \\
|t-s| \leq 1 / n}}\left|\beta_{n}(t)-\beta_{n}(s)\right|=O_{\mathbb{P}}\left(n^{-1} \log n\right) .
\end{aligned}
$$

Fix $t \in[0,1]$ and $\lambda \in[0,1-t]$, then set $x=Q(t)+h$, for $h>0$. By combining the definition (2) of $R_{\lambda}(\cdot)$ with the relation $\mathbb{F}(Q(t))=t$, following from (16), we get

$$
\begin{aligned}
R_{\lambda}(Q(t)) & =\inf \{h>0: \mathbb{F}(Q(t)+h)-\mathbb{F}(Q(t)) \geq \lambda\} \\
& =\inf \{x>0: \mathbb{F}(x) \geq t+\lambda\}-Q(t) \\
& =Q(t+\lambda)-Q(t)=\lambda .
\end{aligned}
$$

We infer from the above assertions (26), (27) and (28), that, as $n \rightarrow \infty$,

$$
\begin{aligned}
& \sup _{\lambda \in \mathcal{H}_{n}}\left|\left\{\frac{n \lambda}{2 \log _{+}(1 / \lambda)}\right\}^{1 / 2} \sup _{t \in[0,1-\lambda]} \pm\left\{\frac{R_{n, \lambda}(Q(t))-R_{\lambda}(Q(t))}{\lambda}\right\}-1\right| \\
& =\sup _{\lambda \in \mathcal{H}_{n}}\left|\left\{\frac{n \lambda}{2 \log _{+}(1 / \lambda)}\right\}^{1 / 2} \sup _{t \in[0,1-\lambda]} \pm\left\{\frac{R_{n, \lambda}(t)-\lambda}{\lambda}\right\}-1\right| \\
& \leq \sup _{\lambda \in \mathcal{H}_{n}}\left|\sup _{t \in[0,1-\lambda]} \pm\left\{\frac{\beta_{n}(t+\lambda)-\beta_{n}(t)}{\sqrt{2 \lambda \log _{+}(1 / \lambda)}}\right\}-1\right|+\sup _{\lambda \in \mathcal{H}_{n}}\left\{\frac{1}{\sqrt{2 \lambda \log _{+}(1 / \lambda)}}\right\} \times O_{\mathbb{P}}\left(n^{-1 / 2} \log n\right),
\end{aligned}
$$

which, by the limit law (14) and the condition (5), equals

$$
O_{\mathbb{P}}\left(\left\{\frac{\log n}{n a_{n}}\right\}^{1 / 2}\right)=o_{\mathbb{P}}(1) .
$$

This yields (24), as sought. $\square$ 
Lemma 2 Whenever $\mathcal{H}_{n}=\left[a_{n}, b_{n}\right]$ satisfies condition (5) of Theorem 1, we have, as $n \rightarrow \infty$,

$$
\sup _{\lambda \in \mathcal{H}_{n}}\left|\sup _{t \in[0,1-\lambda]} \pm\left\{\frac{R_{n, \lambda}(Q(t)) R_{\lambda}(Q(t))}{\lambda^{2}}-1\right\}-\left\{\frac{2 \log _{+}(1 / \lambda)}{n \lambda}\right\}^{1 / 2}\right|=o_{\mathbb{P}}(1) .
$$

Proof. We make use of (28), and of the assumption that $Q(t)=t, t \in[0,1]$. We so see that $R_{\lambda}(Q(t))=Q(t+\lambda)-Q(t)=\lambda$ for $t \in[0,1-\lambda]$, whence

$$
\begin{aligned}
& \sup _{t \in[0,1-\lambda]} \pm\left\{\frac{R_{n, \lambda}(Q(t)) R_{\lambda}(Q(t))}{\lambda^{2}}-1\right\}=\sup _{t \in[0,1-\lambda]} \pm\left\{\frac{R_{n, \lambda}(Q(t))}{R_{\lambda}(Q(t))}-1\right\} \\
& =\sup _{t \in[0,1-\lambda]} \pm\left\{\frac{R_{n, \lambda}(Q(t))-R_{\lambda}(Q(t))}{\lambda}\right\}
\end{aligned}
$$

By combining these equalities with (24) of Lemma 1, we obtain (30).

Proof of Theorem 1 - I The observation (23), together with Lemmas 1 and 2, implies (6), with $\mathbb{F}(t)=Q(t)=t$ and $f(t)=q(t)=1$ for $0 \leq t \leq 1$.

\subsection{Proof of Theorem 1 - II}

In this section, we let $\mathbb{F}(\cdot)$ and $Q(\cdot)$ be as in $\S 1$.

Lemma 3 Fix any $0 \leq u<v<1$. Then, whenever $\mathcal{H}_{n}=\left[a_{n}, b_{n}\right]$ satisfies condition (5) of Theorem 1, we have, as $n \rightarrow \infty$,

$$
\sup _{\lambda \in \mathcal{H}_{n}}\left|\left\{\frac{n \lambda}{2 \log _{+}(1 / \lambda)}\right\}^{1 / 2} \sup _{t \in[u, v]} \pm\left\{\frac{\mathbb{V}_{n}\left(\mathbb{U}_{n}(t)+\lambda\right)-(t+\lambda)}{\lambda}\right\}-1\right|=o_{\mathbb{P}}(1) .
$$

Proof. Making use of (22) and the definition (12) of $\beta_{n}(\cdot)$, we observe that, for each $\lambda>0$ and $t \in[u, v]$,

$$
\mathbb{V}_{n}\left(\mathbb{U}_{n}(t)+\lambda\right)-(t+\lambda)=n^{-1 / 2}\left\{\beta_{n}\left(\mathbb{U}_{n}(t)+\lambda\right)-\beta_{n}\left(\mathbb{U}_{n}(t)\right)\right\}+O_{\mathbb{P}}\left(n^{-1} \log n\right) .
$$

We next infer from (27) of Lemma 1, when combined with the limit law (14) of Fact 1 and condition (5), that, as $n \rightarrow \infty,(31)$ holds.

We shall need the following variant of Lemma 1.

Lemma 4 Whenever $\mathcal{H}_{n}=\left[a_{n}, b_{n}\right]$ satisfies condition (5) of Theorem 1, we have, as $n \rightarrow \infty$,

$$
\sup _{\lambda \in \mathcal{H}_{n}}\left|\left\{\frac{n \lambda}{2 \log _{+}(1 / \lambda)}\right\}^{1 / 2} \sup _{t \in[0,1-\lambda]} \pm\left\{\frac{R_{n, \lambda}(Q(t))-R_{\lambda}(Q(t))}{\lambda q(t)}\right\}-1\right|=o_{\mathbb{P}}(1) .
$$

Proof. Letting $\lambda>0$, and $t \in[0,1-\lambda]$, we combine the definitions (1)-(28) of $R_{n, \lambda}(\cdot)$ and of $R_{\lambda}(Q(t))$, with the relations $\mathbb{F}(Q(t))=t, Q_{n}(t)=Q\left(\mathbb{V}_{n}(t)\right)$ and $\mathbb{F}_{n}(x)=$ $\mathbb{U}_{n}(\mathbb{F}(x))$, for $x \in \mathbb{R}$, following respectively from (16), (17) and (18), to see that,

$$
\begin{aligned}
R_{n, \lambda}(Q(t))-R_{\lambda}(Q(t))= & \inf \left\{h>0: \mathbb{F}_{n}(Q(t)+h)-\mathbb{F}_{n}(Q(t)) \geq \lambda\right\} \\
& -\{Q(t+\lambda)-Q(t)\} \\
= & \left\{Q_{n}\left(\mathbb{F}_{n}(Q(t))+\lambda\right)-Q(t)\right\} \\
& -\{Q(t+\lambda)-Q(t)\} \\
= & Q\left(\mathbb{V}_{n}\left(\mathbb{U}_{n}(F(Q(t)))+\lambda\right)-Q(t+\lambda)\right. \\
= & Q\left(\mathbb{V}_{n}\left(\mathbb{U}_{n}(t)+\lambda\right)\right)-Q(t+\lambda) .
\end{aligned}
$$


Fix an $\varepsilon$, such that $0<\varepsilon<q\left(u^{\prime}\right)$ and select $0 \leq u^{\prime}<u<v<v^{\prime} \leq 1$, in such a way that

$$
\sup _{s, t \in\left[u^{\prime}, v^{\prime}\right]}\left|\frac{q(t)}{q(s)}-1\right|<\varepsilon .
$$

If we let $\lambda$ vary in $\mathcal{H}_{n}=\left[a_{n}, b_{n}\right]$, there exists a.s., an $n_{0}$ such that, for all $n \geq n_{0}$ and $t \in[u, v]$, we have $t+\lambda \in\left[u^{\prime}, v^{\prime}\right]$. Moreover, it is readily checked that, if we denote by $\mathcal{A}_{n}$ the event

$$
\mathcal{A}_{n}=\left\{\mathbb{V}_{n}\left(\mathbb{U}_{n}(t)+\lambda\right) \in\left[u^{\prime}, v^{\prime}\right]: \forall t \in[u, v] \text { and } \lambda \in \mathcal{H}_{n}\right\},
$$

then, $\mathbb{P}\left(\mathcal{A}_{n}\right) \rightarrow 1$ as $n \rightarrow \infty$. This, in turn, implies that, on the event $\mathcal{A}_{n}$, we have, for all $t \in[u, v]$ and $\lambda \in \mathcal{H}_{n}$, as $n \rightarrow \infty$,

$$
\begin{aligned}
& (1-\varepsilon) q(t)\left\{\frac{\mathbb{V}_{n}\left(\mathbb{U}_{n}(t)+\lambda\right)-(t+\lambda)}{\lambda}\right\} \\
\leq & \frac{Q\left(\mathbb{V}_{n}\left(\mathbb{U}_{n}(t)+\lambda\right)\right)-Q(t+\lambda)}{\lambda} \\
\leq & (1+\varepsilon) q(t)\left\{\frac{\mathbb{V}_{n}\left(\mathbb{U}_{n}(t)+\lambda\right)-(t+\lambda)}{\lambda}\right\} .
\end{aligned}
$$

In view of (31), (33) and (35), we so obtain that, as $n \rightarrow \infty$,

$$
\mathbb{P}\left(\sup _{\lambda \in \mathcal{H}_{n}}\left|\left\{\frac{n \lambda}{2 \log _{+}(1 / \lambda)}\right\}^{1 / 2} \times \sup _{t \in[u, v]} \pm\left\{\frac{R_{n, \lambda}(Q(t))-R_{\lambda}(Q(t))}{\lambda q(t)}\right\}-1\right|>\varepsilon\right) \rightarrow 0 .
$$

Given this last statement, we conclude readily (32) by splitting $[0,1]$ into a finite union of intervals $\left[u^{\prime}, v^{\prime}\right]$ on which the oscillation of $q(\cdot)$ fulfills $(34)$, and then, by choosing $\varepsilon>0$ arbitrarily small.

We shall also need the following variant of Lemma 2.

Lemma 5 Whenever $\mathcal{H}_{n}=\left[a_{n}, b_{n}\right]$ satisfies condition (5) of Theorem 1, we have, as $n \rightarrow \infty$,

$$
\sup _{\lambda \in \mathcal{H}_{n}}\left|\sup _{t \in[0,1-\lambda]} \pm\left\{\frac{R_{n, \lambda}(Q(t)) R_{\lambda}(Q(t))}{\lambda^{2} q(t)^{2}}-1\right\}-\left\{\frac{2 \log _{+}(1 / \lambda)}{n \lambda}\right\}^{1 / 2}\right|=o_{\mathbb{P}}(1) .
$$

Proof. The proof is essentially identical to the proof of Lemma 2, adding the following observation. Making use of the definitions (3)-(15) of $M_{\lambda}(\cdot)$ and of $q(\cdot)$, we see that, uniformly over $t \in(0,1]$, we have, as $\lambda \rightarrow 0$,

$$
\lambda q(t) / R_{\lambda}(Q(t)) \rightarrow 1
$$

We omit details.

Proof of Theorem 1. We have now in hand all the necessary ingredients to prove Theorem 1. In view of the definitions (3)-(4)-(15) of $M_{\lambda}(\cdot), f_{n, \lambda}(\cdot)$ and of $q(\cdot)$, we write,

$$
\frac{f_{n, \lambda}(Q(t))-M_{\lambda}(Q(t))}{f(Q(t))}=-\frac{\lambda^{2} q(t)^{2}}{R_{n, \lambda}(Q(t)) R_{\lambda}(Q(t))}\left\{\frac{R_{n, \lambda}(Q(t))-R_{\lambda}(Q(t))}{\lambda q(t)}\right\} .
$$

This, when combined with Lemmas 4 and 5, readily implies (6). The proof of Theorem 1 is therefore completed. 


\section{References}

[1] Beck, J. (1979). The exponential rate of convergence of error for $k_{n}$-NN nonparametric regression and decision. Problems Control Inform. Theory/Problemy Upravlen. Teor. Inform. 8, 303-311.

[2] Berlinet, A. and Devroye, L. A. (1994). Comparison of kernel density estimates. Publ. Inst. Statist. Univ. Paris $38,3-59$.

[3] Burba, F., Ferraty, F. and Vieu, P. (2009). k-nearest neighbour method in functional nonparametric regression. J. Nonparametr. Stat. 21, 453-469.

[4] Collomb, G. (1980). Estimation de la régression par la méthode des $k$ points les plus proches avec noyau : quelques propriétés de convergence ponctuelle. Lecture Notes in Mathematics 821, Springer-Verlag, 159-175.

[5] Csörgő and M.; Révész, P. (1980). A preliminary version of the preliminary version of an invariance principle for N. N. empirical density functions. Carleton Mathematical Lecture Note 27, 91-113.

[6] Deheuvels, P. (2011). One bootstrap suffices to generate sharp uniform bounds in functional estimation. Kybernetica 47, 881-891.

[7] Deheuvels, P. and Derzko, G. (2008) Asymptotic certainty bands for kernel density estimators based upon a bootstrap resampling scheme. Statistical models and methods for biomedical and technical systems. 171-186, Stat. Ind. Technol., Birkhäuser Boston, Boston, MA.

[8] Deheuvels, P. and Devroye, L. (1984). Strong laws for the maximal $k$-spacing when $k \leq c \log n$. Z. Wahrscheinlichkeit. verw. Gebiete. 66, 315-334.

[9] Deheuvels, P. and Mason, D. M. (1992). Functional laws of the iterated logarithm for the increments of empirical and quantile processes. Ann. Probab. 20, 1248-1287.

[10] Deheuvels, P. and Mason, D. M. (2004). General asymptotic confidence bands based on kernel-type function estimators. Statist. Infer. Stoch. Processes. 7, 225-277.

[11] Deheuvels, P. and Ouadah, S. (2011). Uniform in bandwidth functionnal limit laws. J. Theor. Probab., published online (DOI : 10.1007/s10959-011-0376-1).

[12] del Barrio, E., Deheuvels, P. and van de Geer, S. (2007) Lectures on empirical processes. Theory and statistical applications. EMS Series of Lectures in Mathematics.

[13] Devroye, L. (1982). Necessary and sufficient conditions for the pointwise convergence of nearest neighbor regression function estimates. Z. Wahrsch. Verv. Gebiete 61, 467481.

[14] Devroye, L.P. and Wagner, T. J. (1977). The strong uniform consistency of nearest neighbor density estimates. Ann. Statist. 5, 536-540.

[15] Dvoretzky, A., Kiefer, J. and Wolfowitz, J. (1956). Asymptotic minimax character of the sample distribution function and of the classical multinomial estimator. Ann. Math. Statist. 27, 642-669. 
[16] Einmahl, U. and Mason, D. M. (2005). Uniform in bandwidth consistency of kerneltype function estimators. Ann. Statist. 33, 1380-1403.

[17] Fix, E. and Hodges, J.L.(1951). Discriminatory analysis. Nonparametric discrimination: consistency properties. Technical Report 4, Project no. 21-49-004, USAF School of Aviation Medicine, Randoff Fiels, Texas.

[18] Loftsgaarden, D. O. and Quesenberry, C. P. (1965) A nonparametric estimate of a multivariate density function. Ann. Math. Statist. 36, 1049-1051.

[19] Mack, Y. P. (1983) Rate of strong uniform convergence of $k$-NN density estimates. J. Statist. Plann. Inference 8, 185-192.

[20] Moore, D. S. and Henrichon, E. G. (1969). Uniform consistency of some estimates of a density function. Ann. Math. Statist. 40, 1499-1502.

[21] Ralescu, S. S. (1995). The law of the iterated logarithm for the multivariate nearest neighbor density estimators. J. Multivariate Anal. 53, no. 1, 159-179.

[22] Shorack, G. R. (1982). Kiefer's theorem via the Hungarian construction. Z. Wahrscheinlichkeit. verw. Gebiete. 61, 369-373.

[23] Viallon, V. (2007). Functional limit laws for the increments of the quantile process; with applications. Electron. J. Stat. 1,496-518. 